\title{
Correlations between Phlegm Syndrome of Chinese Medicine and Coronary Angiography: A Systematic Review and Meta-Analysis
}

\author{
Qiu-Yan Zhang, ${ }^{1}$ Hao Liang, ${ }^{1,2}$ Hou-Wu Gong, ${ }^{1}$ Hui-yong Huang, \\ Xiao-qing Zhou, ${ }^{1}$ and Xiang Sun $^{3}$ \\ ${ }^{1}$ Hunan University of Chinese Medicine, Science-Education Industrial Park, Yuelu Region, Changsha 410208, China \\ ${ }^{2}$ The First Hospital of Hunan University of Chinese Medicine, No. 95 of Shaoshan Road, Yuhua Region, Changsha 410007, China \\ ${ }^{3}$ No. 8 Hospital of Changsha, No. 22 of Xingsha Street, Xingsha Region, Changsha 410100, China
}

Correspondence should be addressed to Hao Liang; lianghao118@gmail.com

Received 21 August 2014; Revised 18 September 2014; Accepted 23 October 2014

Academic Editor: Ka-Fai Chung

Copyright (c) 2015 Qiu-Yan Zhang et al. This is an open access article distributed under the Creative Commons Attribution License, which permits unrestricted use, distribution, and reproduction in any medium, provided the original work is properly cited.

\begin{abstract}
Phlegm is one of the most common patterns of coronary artery disease (CAD) in Chinese medicine. Our research was aimed at investigating the association between phlegm syndrome of CAD and coronary angiography (CAG) by meta-analysis. According to inclusion criteria, a total of 30 studies involving 5,055 CAD patients were included. The meta-analysis showed that phlegm syndrome patients were prone to multivessel disease ( 28 studies, $\mathrm{OR}=1.53,95 \% \mathrm{CI}, 1.24$ to $1.88, P<0.01$ ) and higher Gensini score ( 2 studies, $\mathrm{OR}=5.90,95 \% \mathrm{CI}, 1.86$ to $9.94, P=0.004)$, but not obviously relevant to severe stenosis $(\geq 75 \%)$ of coronary arteries $(13$ studies, $\mathrm{OR}=1.20,95 \% \mathrm{CI}, 0.63$ to $2.27, P=0.57$ ). We concluded that the coronary arteries lesions of CAD patients with phlegm syndrome were more severe than those with nonphlegm syndromes. Phlegm syndrome should, therefore, be regarded as a dangerous pattern of $\mathrm{CAD}$ with worse prognosis.
\end{abstract}

\section{Introduction}

Coronary artery disease (CAD) is a prevalent disease against human health, dedicated to one of the leading causes of death [1]. Traditional Chinese medicine (TCM) has fought against $\mathrm{CAD}$ (belonging to the categories of Xiong-bi and cardialgia in TCM) for thousands of years, establishing unique theories for etiology and systems of diagnosis and treatment. The syndrome (also called pattern [2]) and syndrome differentiation are the comprehensive analysis of clinical information and can be deemed to be the TCM theoretical interpretation of the symptom profiles [3]. Syndrome differentiation can be used for further stratification of the patients' conditions with certain disease, identified by orthodox medical diagnosis. It guides the choice of treatment either by acupuncture or by TCM herbal formulae and helps in the improvement of efficacy of the selected intervention [4].
Phlegm and blood-stasis are regarded as the most common patterns of CAD patients [5]. They are significantly related to hyperlipidemia [6] and atherosclerosis and platelet activating system [7], which determines the prognosis and stratification of CAD. Phlegm is defined as a viscous, turbid pathological product that can accumulate in the body, causing a variety of diseases [2]. TCM has two general categories of phlegm: broadly defined phlegm and narrowly defined phlegm (visible phlegm such as nasal discharge or sputum from respiratory passages). If the former, invisible phlegm accumulated in the chest and blocked the channels and vessels of heart, it would lead to the so-called phlegm syndrome of Xiong-bi characterized as choking or crushing chest discomfort, shortness of breath, heavy feeling in the limbs, gastric stuffiness, sticky slimy sensation in the mouth, slimy and thick tongue coating, and slippery pulse [8], which was similar to manifestations of typical angina. 
Lots of studies about the correlations between TCM syndromes and coronary angiography (CAG) were published recently, providing new objective information for syndrome differentiation of CAD [9]. Our previous pooled analysis showed a strong relation between blood-stasis syndrome and CAG [10], but the relevance of phlegm syndrome to presentations of CAG was not explored. This study was to investigate whether the phlegm syndrome was related to CAG through a systematic review and meta-analysis.

\section{Methods}

2.1. Search Strategy. MOOSE (Meta-Analysis of Observational Studies in Epidemiology) and PRISMA (Preferred Reporting Items for Systematic Reviews and Meta-Analyses) statements were carefully consulted [41, 42]. To identify all relevant studies, we performed a literature search (Chinese and English languages) in China Academic Journal Network Publishing Database (CAJD), Chinese Biomedical Literature Database (CBM), China Doctor Dissertation Full-Text Database (CDFD), Chinese Selected Master's Theses FullText Databases (CMFD), and Medline and Embase (January 1990 through June 2014) under strict-making search strategy using Medical Subject Heading (MeSH) term (list of tables, Table 1). We also hand-searched the reference lists of all primary studies and reviews identified by the initial search.

2.2. Study Selection. All diagnostic cross-sectional studies, cohort studies, case-control studies, and randomized studies were retrieved to investigate association between TCM syndromes and presentations of CAG. We included a study if (1) obstructive CAD, with $\geq 50 \%$ diameter stenosis, was selected as the standard for significant $\mathrm{CAD}$, using catheter-based $\mathrm{X}$-ray angiography as the compared standard; (2) reported cases are in absolute numbers that can distinguish between phlegm syndrome and the others; (3) CAG results included at least one of the following parameters: the number of diseased arteries (defined multivessel disease [43] as cases with more than one stenotic ( $\geq 50 \%$ ) diameter coronary artery), degree of coronary artery stenosis (defined severe artery stenosis as $\geq 75 \%$ by CAG), and Gensini score [44] (a common score system for evaluating the culprit coronary vessels). Studies were excluded if (1) animals; (2) review and case report; (3) study focused on specific CAD population, for example, female CAD patients, acute myocardial infarction, CAD with diabetes, and so on (4) duplicate reports.

2.3. Data Extraction and Quality Assessment. Two independent reviewers (Liang and Sun) extracted the following data from the selected studies. Inconsistencies were settled by discussion and consensus. We extracted year of publication, study design, clinical setting, methods of syndrome differentiation, and angiographic parameters. Methodological quality of included primary studies was assessed by two authors (Zhou and Gong) using a modified QUADAS-2 tool that included eight items [45]: patient selection: (1) consecutive or randomized patients; (2) avoiding inappropriate exclusions; (3) inclusion criteria of patients described; syndrome differentiation: (4) syndrome differentiation results interpreted without knowledge of the results of CAG; (5) syndrome differentiation by more than two independent doctors; reference standard: (6) CAG results interpreted without knowledge of the results of the syndrome differentiation; flow and timing: (7) an appropriate interval between syndrome differentiation and CAG; (8) perspective design. They are answered as "yes," "no," or "unclear" and are phrased such that "yes" indicates low risk of bias. Tabular and graphic displays served in summarizing quality assessments.

2.4. Statistical Analysis and Data Synthesis. To identify potential correlations between phlegm syndrome and CAG, we calculated an overall OR with a fixed or random effects model meta-analysis for the indices, which assumes the underlying effect varies according to studies. We performed tests of heterogeneity between studies using a standard chisquare test and $I^{2}$ statistic [46]. To examine sources of heterogeneity, subgroups of studies identified by selected covariates were meta-analyzed separately. Egger's test and Begg's funnel plot were applied for detecting publication bias in the meta-analysis. One-way sensitivity analysis was performed to assess the stability of the results; namely, a single study in the meta-analysis was deleted each time to reflect the influence of the individual data set to the pooled OR [47]. Statistical significance was set at $P<0.05$ and all statistical analyses were performed using RevMan 5.2.2 (The Cochrane Collaboration).

\section{Results}

3.1. Description of Included Studies. The literature process was outlined in Figure 1, and 88 potentially relevant studies were retrieved for detailed evaluation. 58 were excluded because (1) they had overlapping data; (2) it was not possible to calculate absolute numbers from the presented data; (3) or data was inconsistent in the context of the article. Finally, 30 included studies met the inclusion criteria, 29 Chinese articles, and 1 English article. All of the studies were from hospitals of China. Study and population characteristics of the included studies are summarized in Table 2, involving a total of 5,029 patients, reporting age ranging from 37 to 87 years. Analysis of the syndromes showed that there were $1,674(33.3 \%)$ phlegm syndrome patients compared with $3,355(66.7 \%)$ of the others. Most studies reported elective assessment for CAD, with study groups including those with suspected and known CAD. Eight studies were theses from medical universities of China. Methods for syndrome differentiation of CAD were divided into syndrome-element differentiation (SED) and other methods called conventional syndrome differentiation (CSD). CSD contains the classical syndrome differentiation methods, such as viscera syndrome differentiation and eight-principal syndrome differentiation $[2]$.

3.2. Quality Assessment of Included Studies. Table 3 and Figure 2 summarized the quality assessment for the 30 fulltext studies. Quality assessment of most studies was not satisfactory, especially the blinding method. None of the studies for those interpreting syndrome differentiation data 


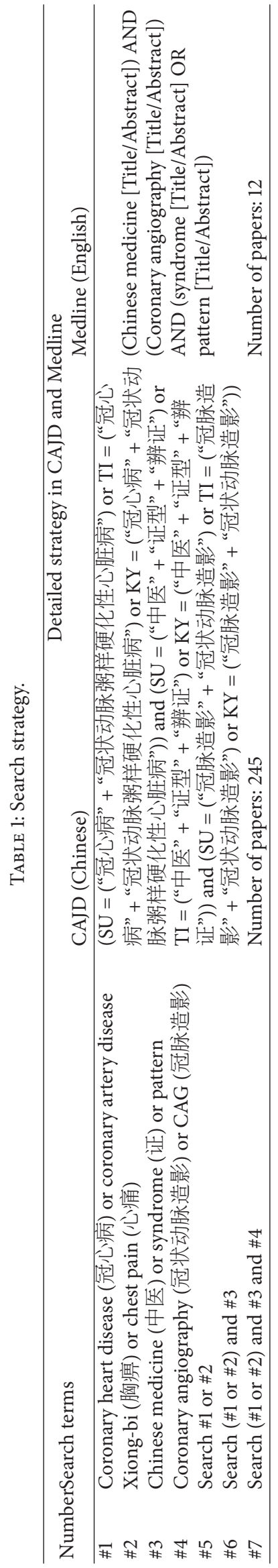




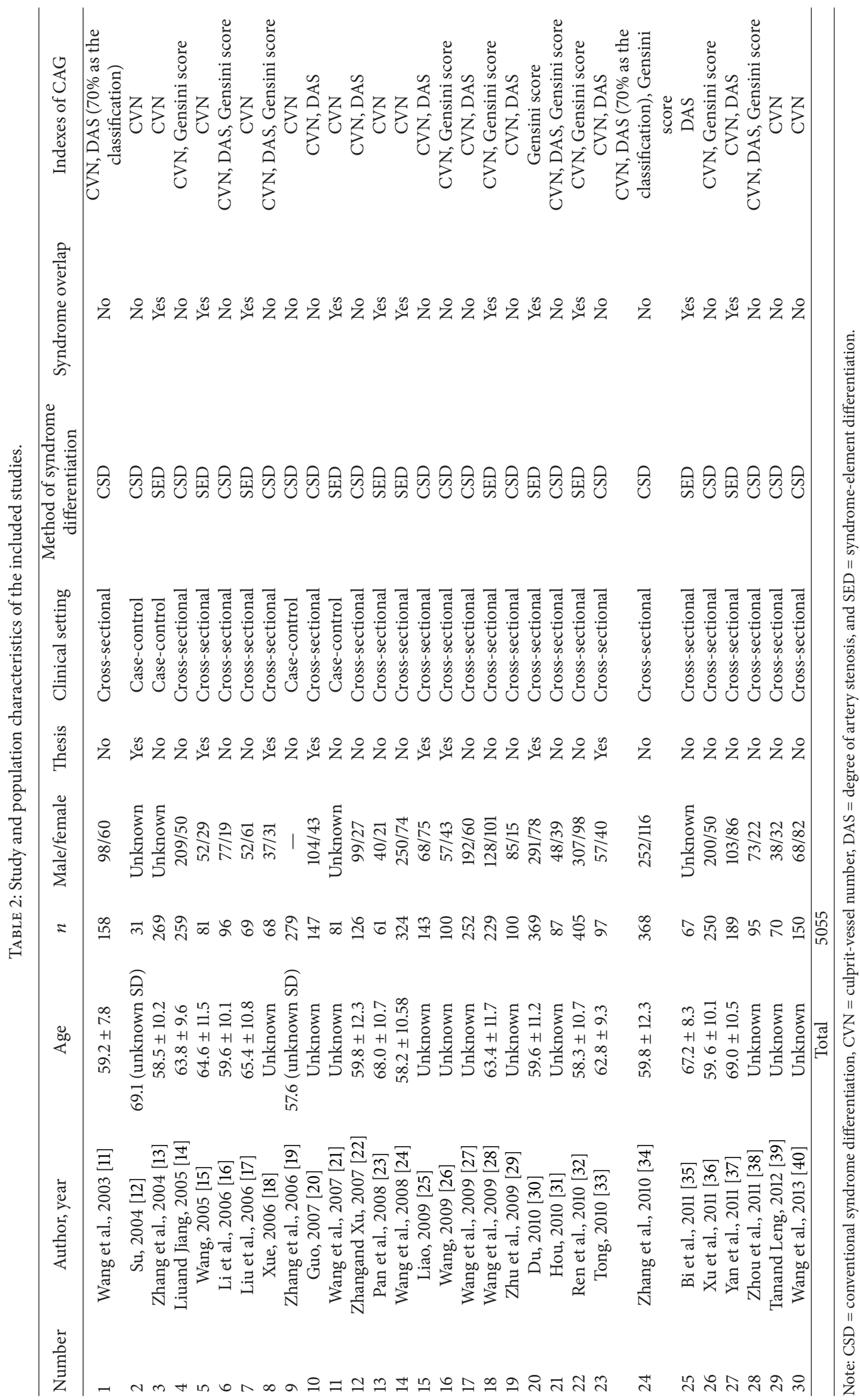




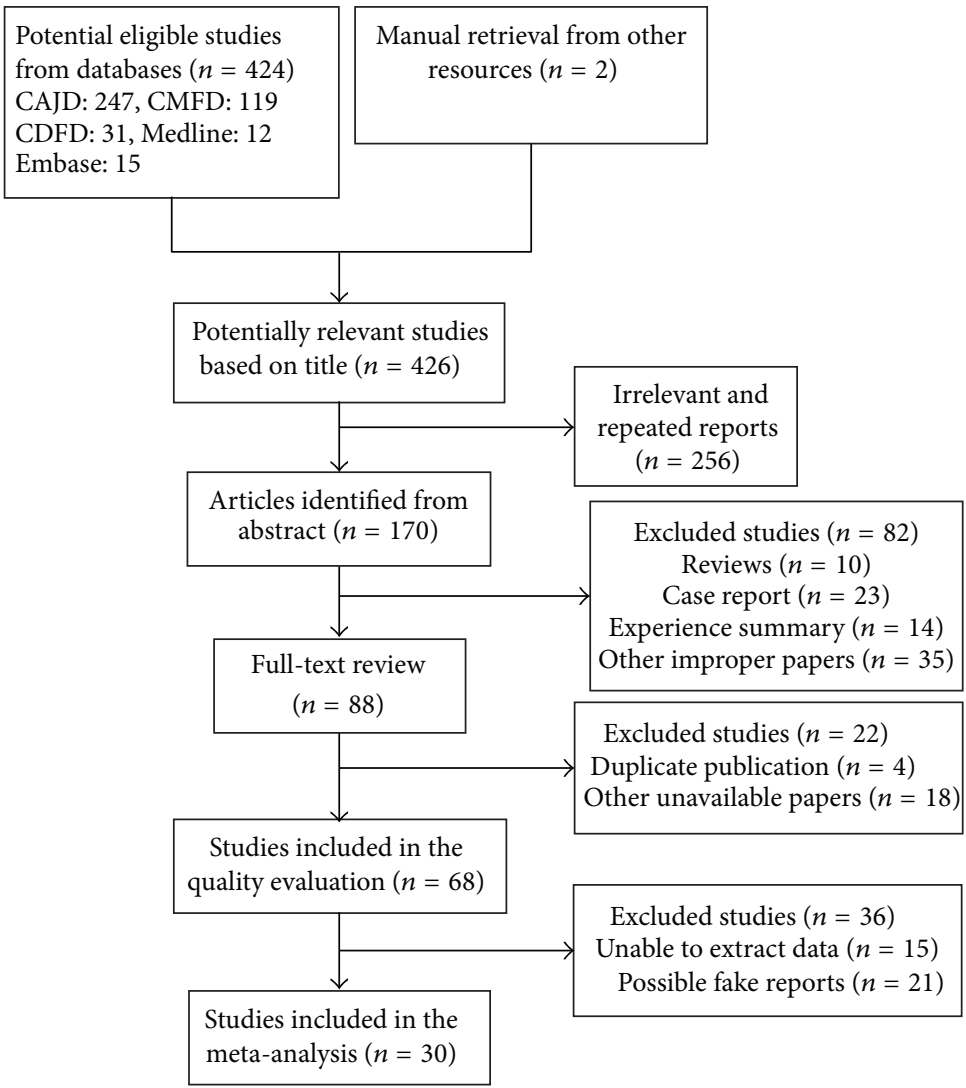

FIgURE 1: Flow diagram of studies considered for the review.

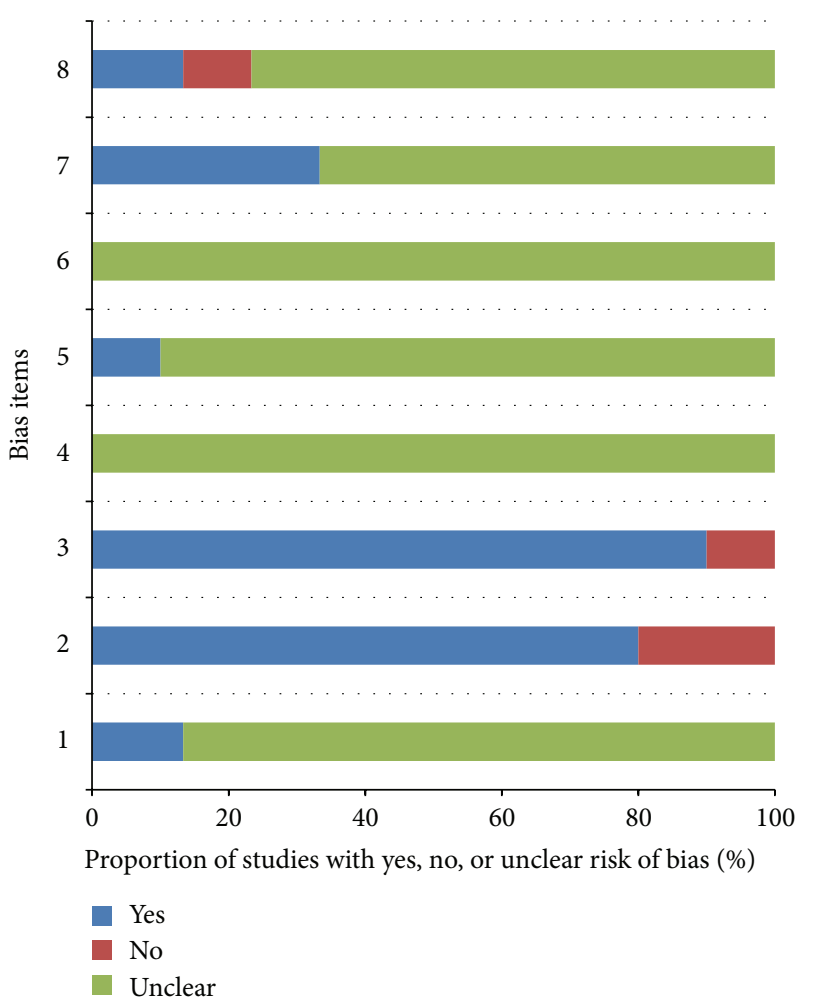

FIGURE 2: Graphic display of quality assessment. Note: (1) consecutive or randomized patients; (2) avoiding inappropriate exclusions; (3) inclusion criteria of patients described; (4) syndrome differentiation results interpreted without knowledge of the results of CAG; (5) syndrome differentiation by more than two independent doctors; (6) CAG results interpreted without knowledge of the results of the syndrome differentiation; (7) an appropriate interval between syndrome differentiation and CAG; (8) perspective design. 
TABLE 3: Tabular display of quality assessment.

\begin{tabular}{|c|c|c|c|c|c|c|c|c|c|}
\hline \multirow{2}{*}{ Number } & \multirow{2}{*}{ Study, year } & \multicolumn{3}{|c|}{ Patient selection } & \multicolumn{2}{|c|}{ Syndrome differentiation } & \multirow{2}{*}{$\begin{array}{c}\text { Reference standard } \\
(6)\end{array}$} & \multicolumn{2}{|c|}{ Flow and timing } \\
\hline & & (1) & $(2)$ & (3) & $(4)$ & $(5)$ & & $(7)$ & $(8)$ \\
\hline 1 & Wang et al., 2003 [11] & Unclear & Yes & Yes & Unclear & Unclear & Unclear & Yes & Unclear \\
\hline 2 & $\mathrm{Su}, 2004[12]$ & Unclear & Yes & Yes & Unclear & Unclear & Unclear & Yes & Unclear \\
\hline 3 & Zhang et al., 2004 [13] & Yes & Yes & Yes & Unclear & Unclear & Unclear & Yes & Yes \\
\hline 4 & Liu and Jiang, 2005 [14] & Unclear & No & Yes & Unclear & Unclear & Unclear & Unclear & Unclear \\
\hline 5 & Wang, 2005 [15] & Yes & Yes & Yes & Unclear & Unclear & Unclear & Unclear & Unclear \\
\hline 6 & Li et al., 2006 [16] & Unclear & Yes & Yes & Unclear & Unclear & Unclear & Unclear & Unclear \\
\hline 7 & Liu et al., 2006 [17] & Unclear & Yes & Yes & Unclear & Unclear & Unclear & Yes & Unclear \\
\hline 8 & Xue, 2006 [18] & Unclear & Yes & Yes & Unclear & Yes & Unclear & Unclear & Unclear \\
\hline 9 & Zhang et al., 2006 [19] & Unclear & Yes & Yes & Unclear & Yes & Unclear & Yes & Yes \\
\hline 10 & Guo, $2007[20]$ & Unclear & No & Yes & Unclear & Unclear & Unclear & Unclear & Unclear \\
\hline 11 & Wang et al., 2007 [21] & Unclear & No & Yes & Unclear & Unclear & Unclear & Yes & Yes \\
\hline 12 & Zhang and $\mathrm{Xu}, 2007$ [22] & Unclear & No & No & Unclear & Unclear & Unclear & Unclear & Unclear \\
\hline 13 & Pan et al., 2008 [23] & Unclear & Yes & Yes & Unclear & Unclear & Unclear & Yes & Unclear \\
\hline 14 & Wang et al., 2008 [24] & Unclear & Yes & Yes & Unclear & Unclear & Unclear & Yes & Unclear \\
\hline 15 & Liao, 2009 [25] & Yes & No & Yes & Unclear & Unclear & Unclear & Yes & No \\
\hline 16 & Wang, 2009 [26] & Unclear & Yes & Yes & Unclear & Yes & Unclear & Unclear & Unclear \\
\hline 17 & Wang et al., 2009 [27] & Unclear & Yes & Yes & Unclear & Unclear & Unclear & Unclear & Yes \\
\hline 18 & Wang et al., 2009 [28] & Unclear & Yes & Yes & Unclear & Unclear & Unclear & Unclear & Unclear \\
\hline 19 & Zhu et al., 2009 [29] & Unclear & Yes & Yes & Unclear & Unclear & Unclear & Unclear & Unclear \\
\hline 20 & $\mathrm{Du}, 2010[30]$ & Unclear & Yes & Yes & Unclear & Unclear & Unclear & Yes & No \\
\hline 21 & Hou, $2010[31]$ & Unclear & Yes & No & Unclear & Unclear & Unclear & Unclear & Unclear \\
\hline 22 & Ren et al., 2010 [32] & Unclear & Yes & Yes & Unclear & Unclear & Unclear & Unclear & Unclear \\
\hline 23 & Tong, $2010[33]$ & Unclear & Yes & Yes & Unclear & Unclear & Unclear & Unclear & No \\
\hline 24 & Zhang et al., 2010 [34] & Unclear & No & No & Unclear & Unclear & Unclear & Unclear & Unclear \\
\hline 25 & Bi et al., 2011 [35] & Yes & Yes & Yes & Unclear & Unclear & Unclear & Unclear & Unclear \\
\hline 26 & Xu et al., 2011 [36] & Unclear & Yes & Yes & Unclear & Unclear & Unclear & Unclear & Unclear \\
\hline 27 & Yan et al., 2011 [37] & Unclear & Yes & Yes & Unclear & Unclear & Unclear & Unclear & Unclear \\
\hline 28 & Zhou et al., 2011 [38] & Unclear & Yes & Yes & Unclear & Unclear & Unclear & Unclear & Unclear \\
\hline 29 & Tan and Leng, 2012 [39] & Unclear & Yes & Yes & Unclear & Unclear & Unclear & Unclear & Unclear \\
\hline 30 & Wang et al., 2013 [40] & Unclear & Yes & Yes & Unclear & Unclear & Unclear & Unclear & Unclear \\
\hline
\end{tabular}

Note: (1) consecutive or randomized patients; (2) avoiding inappropriate exclusions; (3) inclusion criteria of patients described; syndrome differentiation: (4) syndrome differentiation results interpreted without knowledge of the results of CAG; (5) syndrome differentiation by more than two independent doctors; (6) CAG results interpreted without knowledge of the results of the syndrome differentiation; (7) an appropriate interval between syndrome differentiation and CAG; (8) perspective design.

were blinded to the results of the reference standard test (test review bias avoided) and vice versa (diagnostic review bias avoided).

3.3. Results of Meta-Analysis. After the 30 eligible studies involving 5,055 CAD patients were pooled, there was a significant association between phlegm syndrome and CAG in some parameters. Overall, the multivessel disease OR associated with phlegm syndrome was 1.53 (95\% CI, 1.24 to 1.88, $P<0.01 ; I^{2}=47 \%$, Figure 3 ), while severe artery stenosis was $1.20\left(95 \% \mathrm{CI}, 0.63\right.$ to $2.27, P=0.57 ; I^{2}=$
84\%, Figure 4) and Gensini score was 5.90 (95\% CI, 1.86 to $9.94, P=0.004 ; I^{2}=0 \%$, Figure 5). Although the heterogeneity among the analyzed studies was high, most of the studies referred to a positive association for multivessel disease. As the studies were divided into two subgroups by SED or CSD, the OR of multivessel disease in SED group was $1.34\left(95 \% \mathrm{CI}, 1.08\right.$ to $1.67, P=0.008 ; I^{2}=10 \%$, Figure 3 ), showing that the heterogeneity between the studies decreased substantially compared with the main analysis (Figure 3). For the association between phlegm syndrome and degree of artery stenosis, though the result was negative, the heterogeneity between the analyzed studies of thesis subset 


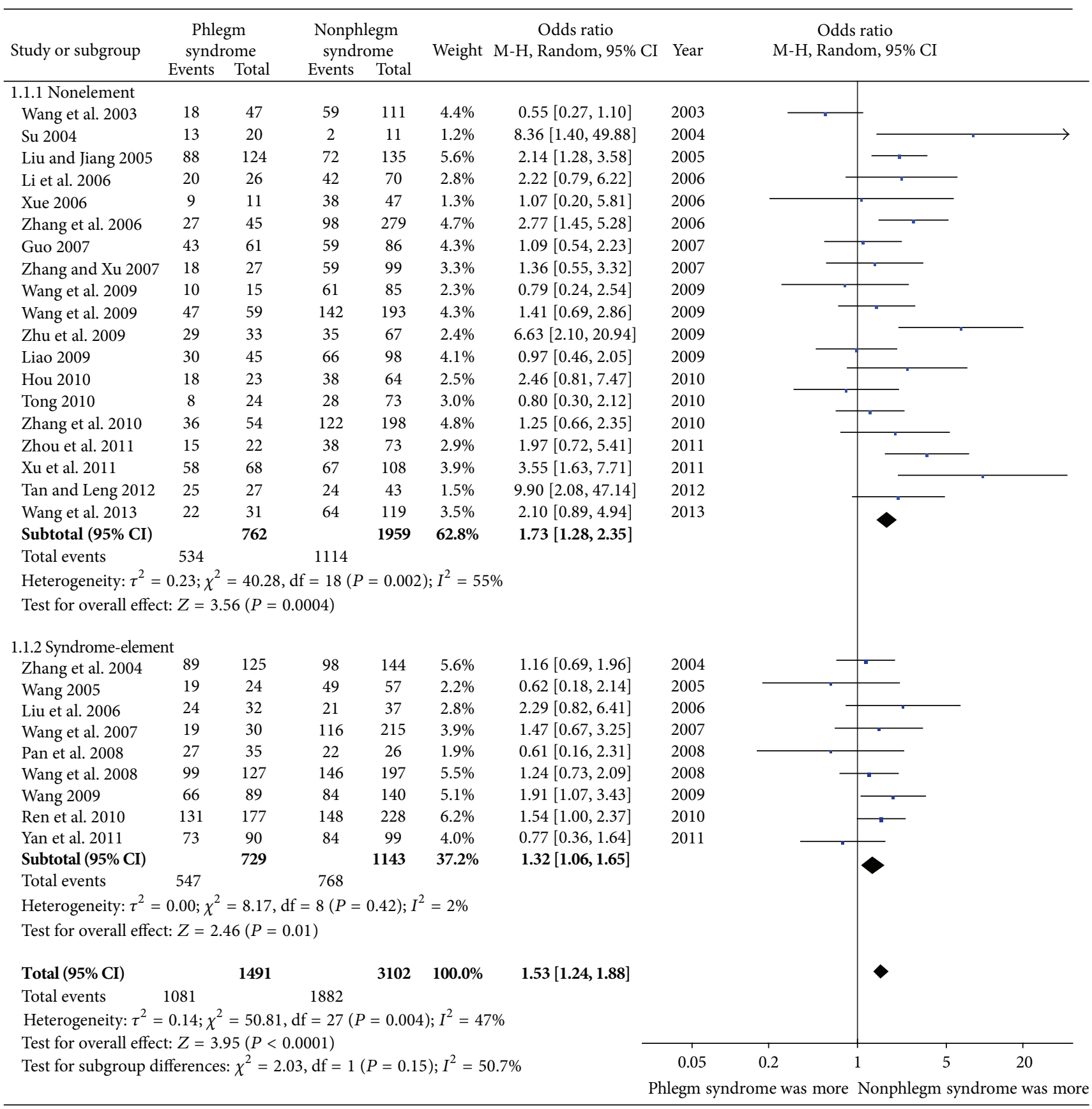

FIGURE 3: Meta-analysis of association between phlegm syndrome and multivessel disease.

sharply reduced $\left(I^{2}=0\right)$ compared with the main analysis (Figure 4).

3.4. Results of Sensitivity Analysis and Publication Bias. No individual studies influencing the summary OR found by one-way sensitivity analysis, indicating that the pooled data was stable. There was no evidence of publication bias, because the funnel plot according to meta-analysis of association between phlegm syndrome and multivessel disease did not show obvious asymmetry by visual inspection (Figure 6), which was also confirmed by Egger's test $(P=0.527)$.
However, some studies located out of the border, which may affect the result of the meta-analysis.

\section{Discussion}

This was the first meta-analysis to address the relationship between phlegm syndrome and CAG. The parameters of culprit vessel, stenosis degree, and Gensini score under CAG were important indicators for CAD patients, and we discovered that serious coronary artery lesions are associated with the presence of phlegm syndrome. The phlegm syndrome 


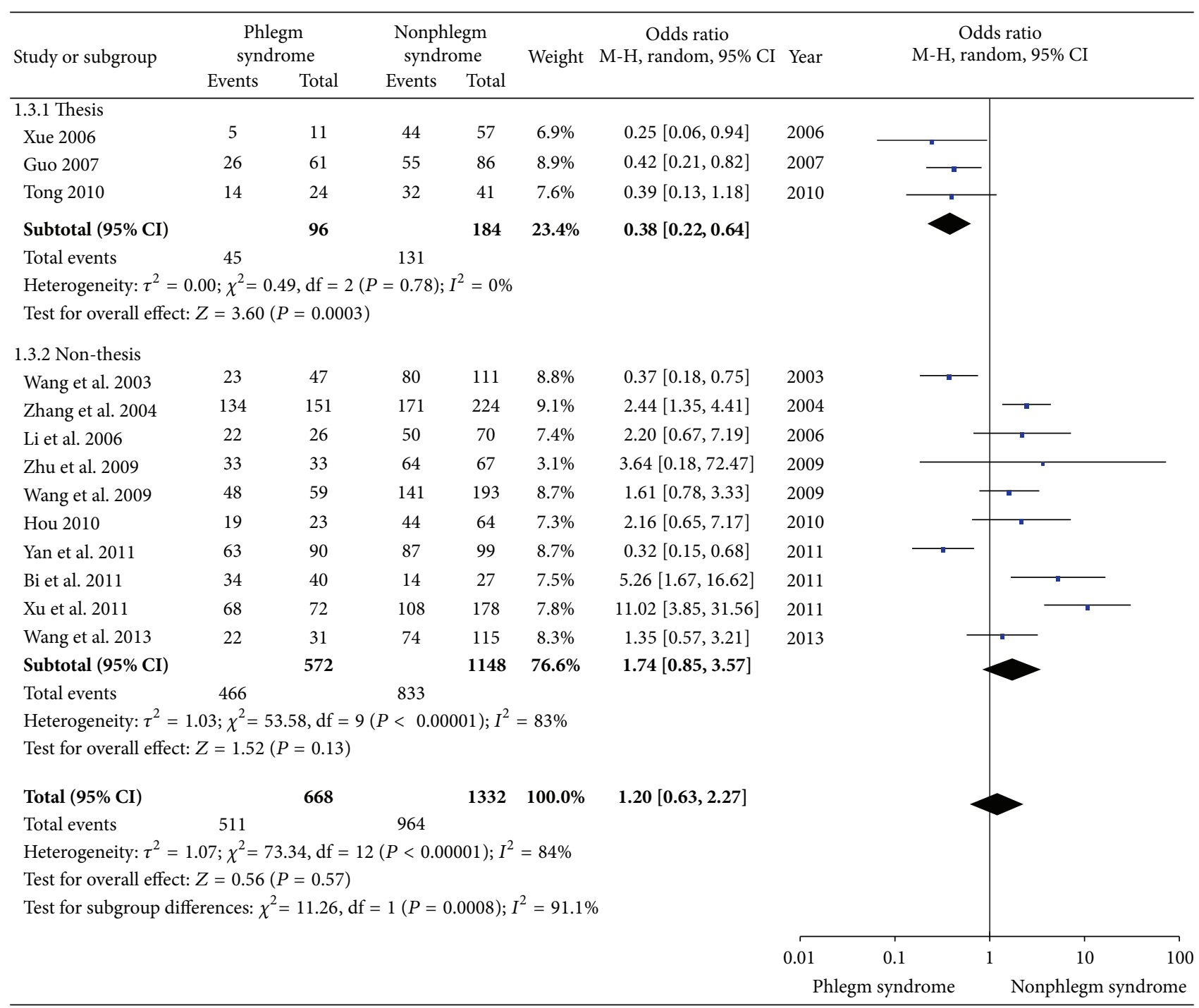

FIGURE 4: Meta-analysis of association between phlegm syndrome and severe artery stenosis.

\begin{tabular}{|c|c|c|c|c|c|c|c|c|c|c|c|c|}
\hline \multirow{3}{*}{$\begin{array}{l}\text { Study or subgroup } \\
\text { Du } 2010\end{array}$} & \multicolumn{2}{|c|}{ Phlegm syndrome } & \multicolumn{3}{|c|}{ Nonphlegm syndrome } & \multirow{2}{*}{ Weight } & \multirow{2}{*}{$\begin{array}{l}\text { Mean difference } \\
\text { IV, fixed, 95\% CI }\end{array}$} & \multirow{2}{*}{ Year } & \multirow{2}{*}{\multicolumn{3}{|c|}{$\begin{array}{l}\text { Mean difference } \\
\text { IV, fixed, } 95 \% \text { CI }\end{array}$}} & \\
\hline & Mean SD & Total & Mean & $\mathrm{SD}$ & Total & & & & & & & \\
\hline & 32.7229 .13 & 143 & 25.24 & 24.28 & 226 & $49.8 \%$ & $7.48[1.75,13.21]$ & 2010 & & & & \\
\hline Ren et al. 2010 & 36.8229 .55 & 177 & 32.49 & 28.38 & 228 & $50.2 \%$ & $4.33[-1.37,10.03]$ & 2010 & & & & \\
\hline \multicolumn{2}{|l|}{ Total $(95 \% \mathrm{CI})$} & 320 & & & 454 & $100.0 \%$ & $5.90[1.86,9.94]$ & & & & & \\
\hline \multirow{2}{*}{\multicolumn{6}{|c|}{$\begin{array}{l}\text { Heterogeneity: } \chi^{2}=0.58, \mathrm{df}=1(P=0.44) ; I^{2}=0 \% \\
\text { Test for overall effect: } Z=2.86(P=0.004)\end{array}$}} & & & -100 & -50 & 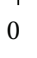 & 50 & 100 \\
\hline & & & & & & & & \multicolumn{2}{|c|}{$\begin{array}{l}\text { Nonphlegm } \\
\text { syndrome high }\end{array}$} & \multicolumn{3}{|c|}{$\begin{array}{l}\text { Phlegm syndrome } \\
\text { high }\end{array}$} \\
\hline
\end{tabular}

FIGURE 5: Meta-analysis of association between phlegm syndrome and Gensini score.

patients were inclined to suffer from multivessel disease and higher Gensini score, but no obvious correlation with the severe artery stenosis was found, which was different from our previous study of meta-analysis for the blood-stasis syndrome [10]. The difference can partly explain the essence of phlegm and blood-stasis syndrome. Blood-stasis and phlegm are both pathological products of abnormal metabolism of body fluid and blood in Chinese medicine. Phlegm formed in the spleen can easily expand and accumulate not only in the lungs but also in almost all other parts of the body, including five viscera, six bowels, joints, and surface tissue, through Meridians or Triple Energizers [48, 49]. Multivessel disease could be considered as diffuse changes of coronary arteries representing the pathological features of phlegm. 


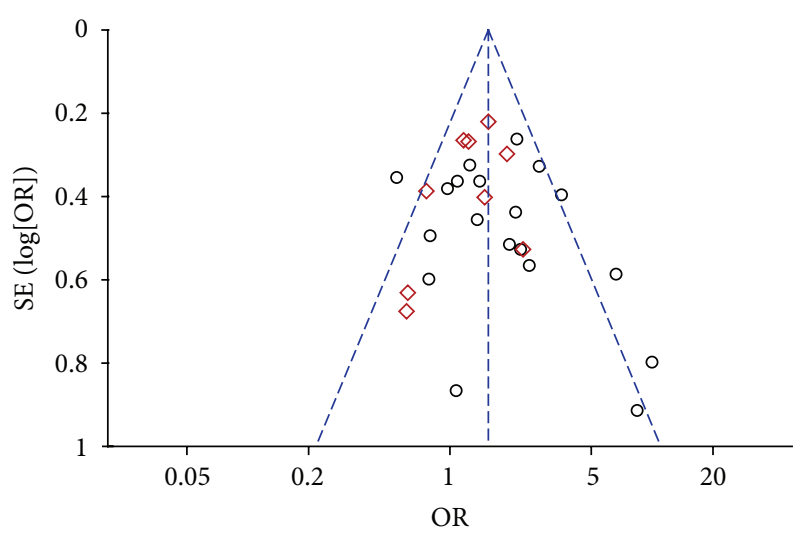

Subgroups

O Non-element

$\diamond$ Syndrome-element

FIGURE 6: Funnel plot for publication bias by association between phlegm syndrome and multivessel disease.

The blood-stasis focused on the "stasis," which means block, retardance, and fixation, so patients of blood-stasis pattern were significantly related to $>75 \%$ degree of artery stenosis [10]. Unfortunately, we were unable to judge which syndrome was worst (phlegm or blood-stasis) for difficulty in extracting the independent data.

Considered as the base of modernization and scientification of TCM, the syndrome diagnostic criteria began to be researched from the 1980s. They were drawn up by different organizations and departments nonrecognized by one another, including Tentative Standard approved by National Academic Seminar on TCM Syndrome Differentiation Based Treatment of CAD in 1980 and Standards for Diagnosis and Curative Effect of TCM Diseases and Syndromes by State Administration of Traditional Chinese Medicine in 1994. Even the process of syndrome differentiation was judged by symptoms and signs with accepted criterion. Plus, the results could be affected by the doctor's learning, medical experience, academic origins, methods, and other factors. Therefore, the judgment made by doctors on cause, location, nature, and trend of disease is individual and variable, and no wonder that 10 TCM doctors may give 10 different diagnoses when facing the same patient [50]. It is crucial, however, to find the source of heterogeneity, usually an obstacle to the meta-analysis. Based on our subgroup analysis, it is strongly supported that different diagnostic methods were the source of heterogeneity and restriction for syndrome differentiation. The heterogeneity of syndrome differentiation based on SED studies decreased to 2\% (Figure 3), while the CSD group increased a little. SED method, now widely used in clinical and basic research, was created by Professor Zhu et al. [51], who proposed the theory of syndrome-element and divided those "elements" into categories of location and nature. The differentiation system was established by clarifying that "syndrome" was composed of the key elements of the location and the nature of disease. Thus, a complete syndrome name was combined by elements of location and nature diagnosed from symptoms and signs. Unlike inflexible CSD, the syndrome could be free combination of possible syndrome-elements, allowing any forms of elements summation. For instance, phlegm is its high affinity with other pathogenic factors such as heat, cold, wind, dampness, and dryness and, because of this, phlegm is often combined with other pathogenic factors to form more complex patterns such as heat-phlegm, coldphlegm, or wind-phlegm [52]. Moreover, our subanalysis proved that SED method led to homogeneity between studies, which would be more promising than CSD. The heterogeneity was also affected by the factor of thesis or not. For postgraduates lack of clinical experience, their diagnosis of syndrome differentiation may be different from older doctors, causing a discrepancy of studies. In a word, the thinking characteristics of syndrome are more similar to physical image thinking rather than image thinking [50]; thus, methods may play an important role during implementation based on the syndrome differentiation criteria.

Any process that yields information used to inform patient management can be regarded as a clinical test [53]. The basic aim of test accuracy studies is to assess how well a test can distinguish between people with and without the disease/syndrome/condition of interest [54]. Nevertheless, unlike index test compared with a reference standard in western medicine, the diagnostic test accuracy (DTA), such as specificity or sensitivity, was still tough for syndrome differentiation just by a profile of symptom combination, or clinical phenotypes. Above all, the design of syndrome differentiation research is similar to diagnostic tests, which means cross-sectional and descriptive in nature [55], and RCT $[56,57]$ case-control and two-gate designs could be feasible [55]. Using QUADAS-2 tool for quality assessment was an innovation of our research. Avoiding subjective impact on researchers, double blind, for instance, is the key to quality of diagnostic study. In the summary of tabular and graphic results, items of (4), (5), and (6) concerning improving objective diagnosis for syndromes were not optimistic (Table 3, Figure 2). It tells us that most of TCM researchers did not take diagnostic design seriously. Lack of blinding can lead to overestimation of test accuracy, especially when the interpretation of test results is subjective [58].

Evaluation score systems of CAG, such as Gensini score [44] and SYNTAX score [59], are more comprehensive and better than just number or degree of stenosis for vessel lesions to estimate conditions of diseased coronary arteries. Due to difficulty of extracting available data, only two studies containing Gensini score were included in the meta-analysis. Moreover, $\mathrm{CAD}$ patients could be subdivided into acute coronary syndrome (ACS) and non-ACS, and ACS attacks suddenly often with worse outcomes than non-ACS. The clinical manifestations and CAG of the two types of patients are quite different [60]. Once mixed up, it would produce more difficulties and confusion for the study. We hope patient spectrum of next researches is aimed at ACS or non-ACS individually. Last but not least, there were no standards or statements specific for publishing meta-analysis of diagnostic tests, so as the TCM syndrome diagnosis. The researchers have to only reference other standards, such as MOOSE [61] 
to finish the review, to some degree limiting the diagnostic research on meta-analysis.

\section{Conclusions}

From the meta-analysis, we found there were correlations between phlegm syndrome of CAD patients and image of CAG: the coronary arteries lesions of CAD patients with phlegm syndrome were more severe than those with other syndromes. Syndrome differentiation was not only important for diagnosis and treatment, but also useful for the prognosis. Phlegm syndrome could be considered as risk syndrome of CAD patients, whom doctors should pay closer attention to. However, from the quality assessment, we found that the design quality of TCM diagnostic test urgently needs to be addressed.

\section{Conflict of Interests}

There is no conflict of interests declared.

\section{Authors' Contribution}

Qiuyan Zhang proposed the idea and drafted the paper. Hao Liang carried out some writing of the paper, performed the meta-analysis by RevMan, and submitted the paper to journals. Houwu Gong participated in the methodological quality evaluation and drew the graphic display of quality assessment. Hui-yong Huang participated in the design of the study and extracted data. Xiaoqing Zhou conceived of the study and participated in quality assessment and helped to draft the paper. All the authors read and approved the final paper.

\section{Acknowledgments}

This study was supported by the Construct Program of the Key Discipline of TCM Diagnostics in Hunan Province (HNPR-2012-03001), Hunan Province TCM Research Funded Project (2006-004), and the State Key Subject of TCM Diagnostics in Hunan University of Chinese Medicine Open Fund (2013ZYZD02).

\section{References}

[1] A. S. Go, D. Mozaffarian, V. L. Roger et al., "Heart disease and stroke statistics-2013 update: a report from the American Heart Association," Circulation, vol. 127, no. 1, pp. e6-e245, 2013.

[2] World Health Organization, WHO International Standard Terminologies on Traditional Medicine in the Western Pacific Region, World Health Organization, Western Pacific Region, 2007.

[3] A. Lu, M. Jiang, C. Zhang, and K. Chan, "An integrative approach of linking traditional Chinese medicine pattern classification and biomedicine diagnosis," Journal of Ethnopharmacology, vol. 141, no. 2, pp. 549-556, 2012.

[4] M. Jiang, C. Lu, C. Zhang et al., "Syndrome differentiation in modern research of traditional Chinese medicine," Journal of Ethnopharmacology, vol. 140, no. 3, pp. 634-642, 2012.
[5] Y. Ren, M. Zhang, K. Chen et al., "Clinical and epidemiological investigation of TCM syndromes of patients with coronary heart disease in China," Evidence-Based Complementary and Alternative Medicine, vol. 2012, Article ID 714517, 5 pages, 2012.

[6] D. Bai and J. Song, "Plasma metabolic biomarkers for syndrome of phlegm and blood stasis in hyperlipidemia and atherosclerosis," Journal of Traditional Chinese Medicine, vol. 32, no. 4, pp. 578-583, 2012.

[7] Y.-L. Wang, D.-Q. An, N. Ma, M. Zhu, and H.-Y. Sun, "Study on the changes of thrombosis-associated factors in patients with coronary heart disease of turbidity-phlegm blocking syndrome," Chinese Journal of Integrative Medicine, vol. 15, no. 5, pp. 337-340, 2009.

[8] State Administration of Traditional Chinese Medicine, Standards for Diagnosis and Curative Effect of TCM Diseases and Syndromes, Nanjing University Publishing House, Nanjing, China, 1994.

[9] Z.-X. Shi and W.-L. Gu, "Exploration of TCM syndrome differentiation of coronary heart disease and coronary angiography," Chinese Journal of Integrative Medicine, vol. 21, no. 1, pp. 76-79, 2007.

[10] X.-Q. Zhou, H. Liang, X. Sun, and H.-T. Zhou, "Correlation between TCM blood stasis pattern of coronary heart disease and coronary angiography result: a meta-analysis," Chinese Journal of Evidence-Based Medicine, vol. 12, no. 12, pp. 1470-1477, 2012.

[11] D.-S. Wang, Z.-K. Yuan, J.-H. Wei, W.-X. Yin, and J.-S. Li, "Study on orrelation between syndrome differentiation of phlegm and blood-stasis syndrome and coronary angiography in coronary heart disease," Journal of Traditional Chinese Medicine, vol. 44, no. 12, pp. 937-938, 2003.

[12] Q. Su, Research on Body Constitution and TCM Syndrome of Coronary Heart Disease and Its Correlation with Objective Indicators, Beijing University of Chinese Medicine, 2004.

[13] M.-Z. Zhang, B.-H. Ding, W.-D. Zhang, J. Li, and C.-S. Ma, "Study on the outcome of coronary angiography and traditional Chinese medicine syndromes in 375 cases of chest stuffiness and pains," Chinese Journal of Integrated Traditional and Western Medicine in Intentire Critical Care, vol. 11, no. 2, pp. 115-117, 2004.

[14] Y.-M. Liu and M.-X. Jiang, "Research on the relationship between 259 patients TCM syndrome differentiation with coronary heart disease and coronary angiographic findings," Beijing Journal of Traditional Chinese Medicine, vol. 24, no. 3, pp. 131-133, 2005.

[15] X.-S. Wang, Study on Correlation Between TCM Syndromes of Coronary Heart Disease and Coronary Angiography, Beijing University of Chinese Medicine, 2005.

[16] J. Li, J.-D. Zhang, and T.-T. Liu, "Relationship between TCM syndrome of coronary heart disease and coronary artery lesion," Journal of Shandong University of Traditional Chinese Medicine, vol. 30, no. 2, pp. 124-126, 2006.

[17] H.-X. Liu, Z.-Y. Wang, W. Peng et al., "Relationship of between angiographic characteristics of 113 patients and TCM syndromes," Journal of China-Japan Friendship Hospital, vol. 20, no. 1, pp. 35-37, 2006.

[18] Z.-M. Xue, Clinical study on relationship between syndrome differentiation type of TCM and coronary angiogram, homocysteine and high sensitivity $C$ reactive protein in patients with coronary heart disease [M.S. thesis], Shandong University of Chinese Medicine, 2006.

[19] G.-Z. Zhang, F.-T. He, F.-W. Wu, J.-T. Bao, and L.-Y. Quan, "Relationship of coronary artery lesions and syndromes differentiation in 415 chest pain cases," China Journal of Traditional 
Chinese Medicine and Pharmacy, vol. 21, no. 8, pp. 498-499, 2006.

[20] D.-M. Guo, Study on the Outcome of Coronary Angiography and Traditional Chinese Medicine Syndrome of Chest Stuffiness and Pains, Xinjiang Medical University, Ürümqi, China, 2007.

[21] J. Wang, Y.-W. Xing, Z.-Z. Li, X.-L. Zhu, and S.-H. Li, “Analysis on pattern characteristic of 102 cases of angina pectoris," Journal of Traditional Chinese Medicine, vol. 48, no. 2, pp. 160-162, 2007.

[22] P. Zhang and W. Xu, "Study on relationship between TCM syndrome type and severity of coronary artery disease," Chinese Journal of Integrative Medicine on Cardio-Cerebrovascular Disease, vol. 5, no. 2, pp. 101-103, 2007.

[23] Z.-Q. Pan, B.-J. Chen, J.-X. Feng et al., "Study of correlation between coronary arteriography and TCM syndrome," Shaanxi Journal of Traditional Chinese Medicine, vol. 29, no. 6, pp. 649651, 2008.

[24] J. Wang, F.-Y. Chu, J. Li et al., "Study on syndrome element characteristics and its correlation with coronary angiography in 324 patients with coronary heart disease," Chinese Journal of Integrative Medicine, vol. 14, no. 4, pp. 274-280, 2008.

[25] J. Liao, The Study on the Relationship between Degree of Coronary Artery Lesion, the Heart Rate Variability and Syndrome Differentiation Type of TCM in Patients with Coronary Heart Disease, Guangzhou University of Chinese Medicine, 2009.

[26] L. Wang, Relationship between the Extent of Coronary Artery Stenosis with the Levels of Hcy and the Syndromes of TCM in Coronary Heart Disease, Fujian University of Traditional Chinese Medicine, 2009.

[27] Z.-K. Wang, Y. Liu, J. Liu, Y. He, B. Li, and Q.-S. Zheng, "Correlation analysis between traditional Chinese medicine syndrome and range and severity of coronary artery lesion in patients with coronary heart disease," Shaanxi Journal of Traditional Chinese Medicine, vol. 30, no. 2, pp. 134-135, 2009.

[28] Z.-Y. Wang, L. Shi, J.-X. Chen et al., "Study on the relationship between TCM syndrome and coronary angiography of patients with coronary artery disease," China Journal of Traditional Chinese Medicine and Pharmacy, vol. 24, no. 9, pp. 1194-1197, 2009.

[29] C.-L. Zhu, K.-P. Yan, M.-J. Zhu et al., "Study on relationship between TCM syndrome of coronary heart disease and coronary angiography," China Journal of Chinese Materia Medica, vol. 34, no. 24, pp. 3289-3291, 2009.

[30] J.-P. Du, The clinical trial of Chinese herbs for supplementing Qi and activating blood circulation on patients with coronary heart disease after percutaneous coronary intervention [Ph.D. thesis], China Academy of Chinese Medical Sciences, 2010.

[31] F.-Q. Hou, "Relationship between coronary artery lesion and TCM syndrome in patients with coronary heart disease," Modern Journal of Integrated Traditional Chinese and Western Medicine, vol. 19, no. 13, pp. 1591-1592, 2010.

[32] Y. Ren, K.-J. Chen, M.-Z. Zhang et al., "Correlation between coronary angiography results and TCM syndromes in 405 patients with coronary heart disease," Journal of Traditional Chinese Medicin, vol. 51, no. 8, pp. 725-728, 2010.

[33] W. Tong, Correlation of coronary artery lesions and TCM syndrome coronary heart disease [M.S. thesis], Beijing University of Chinese Medicine, 2010.

[34] P. Zhang, P. Liu, W.-J. Xu, L.-F. Bi, and W. Wang, "Study on correlation between TCM syndrome and coronary lesion characteristics in 368 coronary heart disease cases," Chinese Journal of Integrative Medicine on Cardio-Cerebrovascular Disease, vol. 8, no. 11, pp. 1308-1311, 2010.
[35] Y.-F. Bi, J.-Y. Mao, Y.-Z. Lu, and X.-L. Wang, "Correlation of traditional Chinese medicine syndrome elements distribution of coronary artery disease with gender and coronary angiography," Chinese Journal of Integrative Medicine on CardioCerebrovascular Disease, vol. 9, no. 9, pp. 1031-1032, 2011.

[36] Y. Xu, J.-Y. Yang, and J. Du, "Study of relationship between TCM syndrome in patients with coronary heart disease and coronary angiography," Gansu Journal of Traditional Chinese Medicine, vol. 24, no. 8, pp. 69-70, 2011.

[37] F. Yan, M.-Z. Zhang, L.-H. Guo et al., "Research of the relativity between TCM syndrome and CAG results in the patients with coronary heart disease," China Journal of Modern Medicine, vol. 21, no. 2, pp. 209-311, 2011.

[38] Y. Zhou, W.-X. Du, M.-D. Zhu, and P. Ma, "Coronary heart disease TCM syndromes and coronary angiography, blood lipid and ejection fraction," Journal of Liaoning University of Traditional Chinese Medicine, vol. 13, no. 6, pp. 78-80, 2011.

[39] Y. Tan and J.-C. Leng, "Correlation of TCM syndromes of coronary heart disease and coronary calcification, coronary angiography," Clinical Journal of Chinese Medicine, vol. 4, no. 21, pp. 15-17, 2012.

[40] Y.-G. Wang, W. Zhong, Y.-W. Yu et al., "Correlation of TCM syndrome with blood lipid and coronary angiographic observation in coronary heart disease," Chinese Journal of Integrative Medicine on Cardio-/Cerebrovascular Disease, vol. 11, no. 7, pp. 770-771, 2013.

[41] D. F. Stroup, J. A. Berlin, S. C. Morton et al., "Meta-analysis of observational studies in epidemiology: a proposal for reporting," The Journal of the American Medical Association, vol. 283, no. 15, pp. 2008-2012, 2000.

[42] D. Moher, A. Liberati, J. Tetzlaff, D. G. Altman, and PRISMA Group, "Preferred reporting items for systematic reviews and meta-analyses: the PRISMA statement," Annals of Internal Medicine, vol. 151, no. 4, pp. 264-269, 2009.

[43] A. E. Rodriguez, C. Fernandez-Pereira, and J. Mieres, "Coronary artery bypass grafting vs percutaneous coronary intervention in multivessel disease," JAMA Internal Medicine, vol. 174, no. 6, p. 1007, 2014.

[44] G. G. Gensini, "A more meaningful scoring system for determining the severity of coronary heart disease," The American Journal of Cardiology, vol. 51, no. 3, p. 606, 1983.

[45] P. F. Whiting, A. W. S. Rutjes, M. E. Westwood et al., "Quadas-2: a revised tool for the quality assessment of diagnostic accuracy studies," Annals of Internal Medicine, vol. 155, no. 8, pp. 529-536, 2011.

[46] J. P. T. Higgins, S. G. Thompson, J. J. Deeks, and D. G. Altman, "Measuring inconsistency in meta-analyses," British Medical Journal, vol. 327, no. 7414, pp. 557-560, 2003.

[47] A. Tobias, "Assessing the influence of a single study in the metaanyalysis estimate," Stata Technical Bulletin, vol. 8, no. 47, 1999.

[48] I. Veith, The Yellow Emperor's Classic of Internal Medicine, University of California Press, Berkeley, Calif, USA, 2002.

[49] S. Clavey, Fluid Physiology and Pathology in Traditional Chinese Medicine, Churchill Livingstone, Edinburgh, UK, 2003.

[50] J. Wang, P. Wang, and X. Xiong, "Current situation and reunderstanding of syndrome and formula syndrome in Chinese medicine," Internal Medicine, vol. 2, no. 3, Article ID 1000113, 2012.

[51] W. F. Zhu, J. F. Yan, and B. Q. Huang, "Application of Bayesian network in syndrome differentiation system of traditional Chinese medicine," Journal of Chinese Integrative Medicine, vol. 4, no. 6, pp. 567-571, 2006. 
[52] Y.-J. Park, J.-S. Park, M.-Y. Kim, and Y.-B. Park, “Development of a valid and reliable phlegm pattern questionnaire," The Journal of Alternative and Complementary Medicine, vol. 17, no. 9, pp. 851-858, 2011.

[53] A. Feinstein, "Misguided efforts and future challenges for research on 'diagnostic tests,' Journal of Epidemiology and Community Health, vol. 56, no. 5, pp. 330-332, 2002.

[54] J. Akers, Systematic Reviews: CRD's Guidance for Undertaking Reviews in Health Care, University of York, Centre for Reviews Dissemination, Heslington, UK, 2009.

[55] A. W. S. Rutjes, J. B. Reitsma, J. P. Vandenbroucke, A. S. Glas, and P. M. M. Bossuyt, "Case-control and two-gate designs in diagnostic accuracy studies," Clinical Chemistry, vol. 51, no. 8, pp. 1335-1341, 2005.

[56] J. G. Lijmer and P. M. M. Bossuyt, "Diagnostic testing and prognosis: the randomised controlled trial in diagnostic research," The Evidence Base of Clinical Diagnosis: Theory and methods of diagnostic research: Second Edition, 2009.

[57] C. J. Biesheuvel, D. E. Grobbee, and K. G. M. Moons, "Distraction from randomization in diagnostic research," Annals of Epidemiology, vol. 16, no. 7, pp. 540-544, 2006.

[58] T. A. Furukawa and G. H. Guyatt, "Sources of bias in diagnostic accuracy studies and the diagnostic process," Canadian Medical Association Journal, vol. 174, no. 4, pp. 481-482, 2006.

[59] G. Sianos, M.-A. Morel, A. P. Kappetein et al., "The SYNTAX Score: an angiographic tool grading the complexity of coronary artery disease," EuroIntervention, vol. 1, no. 2, pp. 219-227, 2005.

[60] D. A. Morrow, C. P. Cannon, R. L. Jesse et al., "National Academy of Clinical Biochemistry Laboratory Medicine Practice Guidelines: clinical characteristics and utilization of biochemical markers in acute coronary syndromes," Clinical Chemistry, vol. 53, no. 4, pp. 552-574, 2007.

[61] T. S. S. Genders, A. Dedic, K. Nieman, and M. G. M. Hunink, "Prognostic value of cardiac computed tomography angiography," Journal of the American College of Cardiology, vol. 57, no. 25, pp. 2543-2544, 2011. 


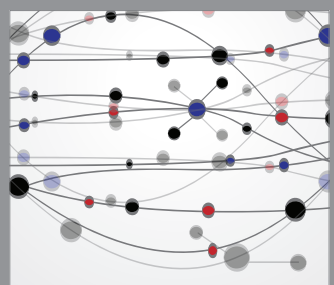

The Scientific World Journal
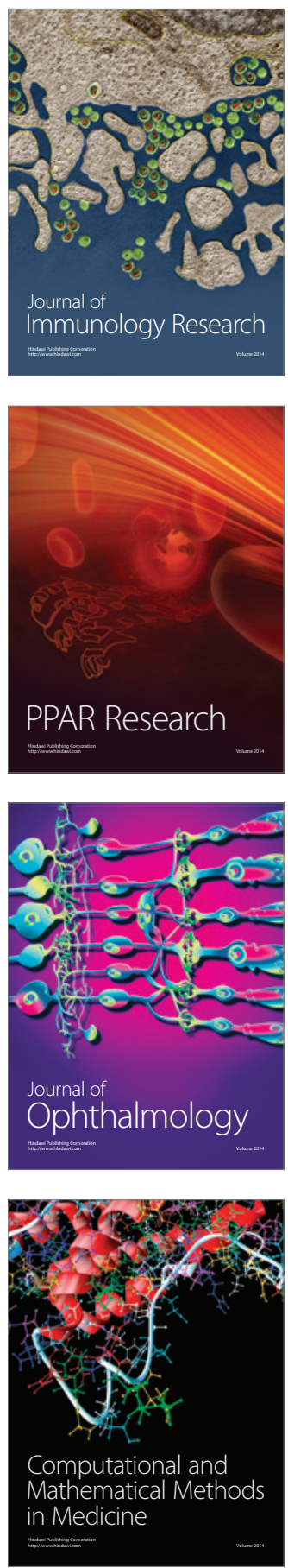

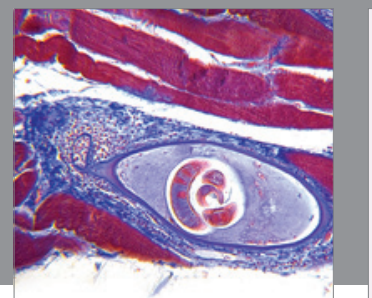

Gastroenterology

Research and Practice
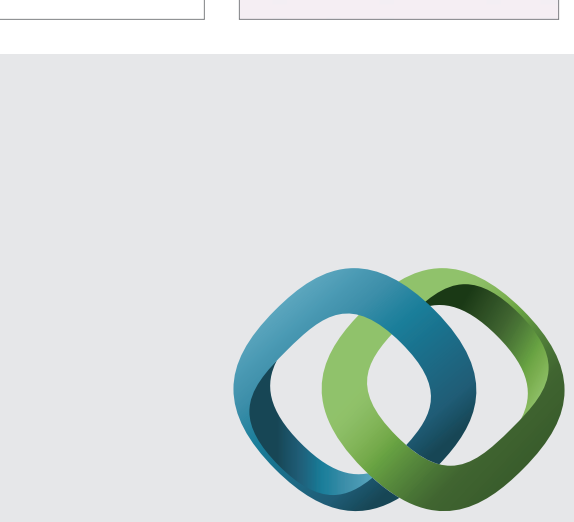

\section{Hindawi}

Submit your manuscripts at

http://www.hindawi.com
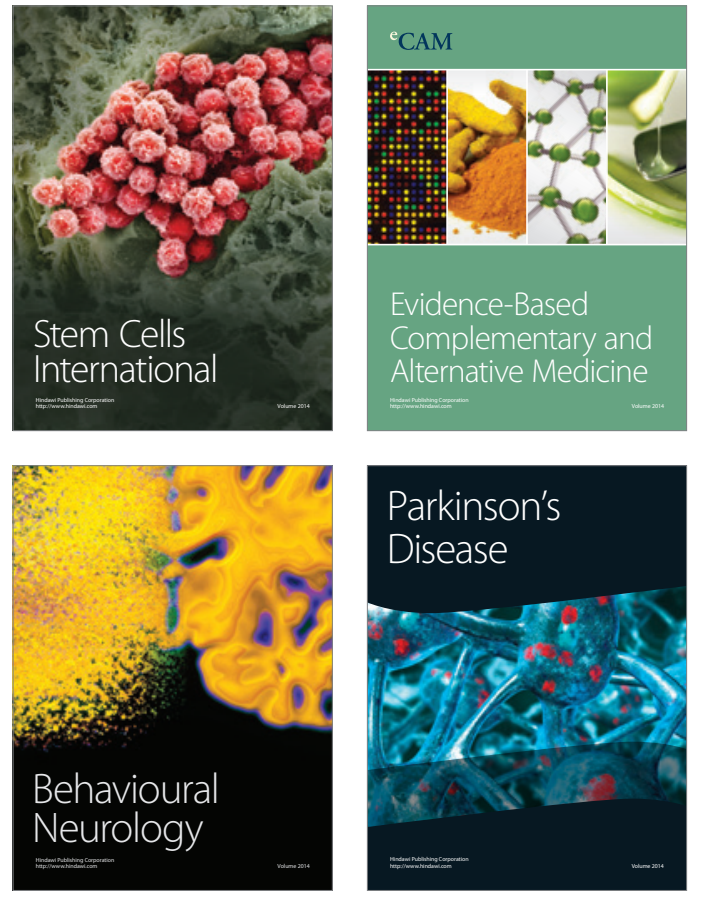
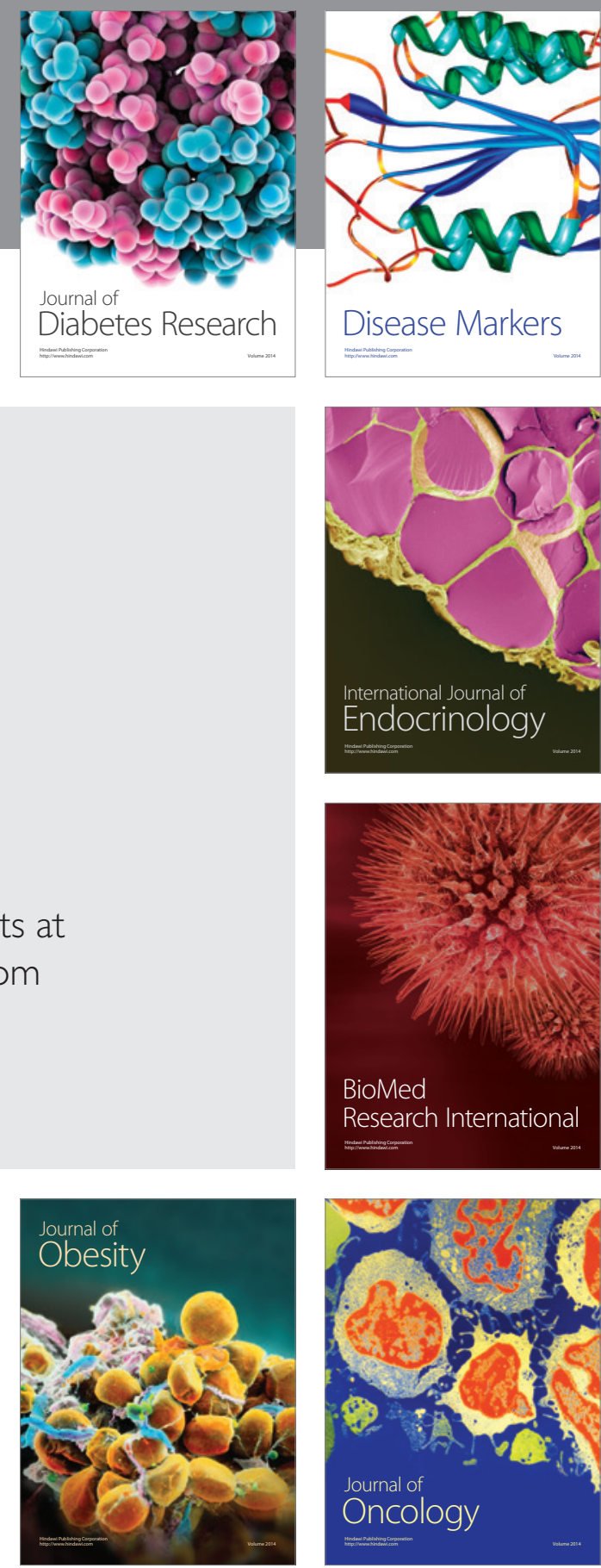

Disease Markers
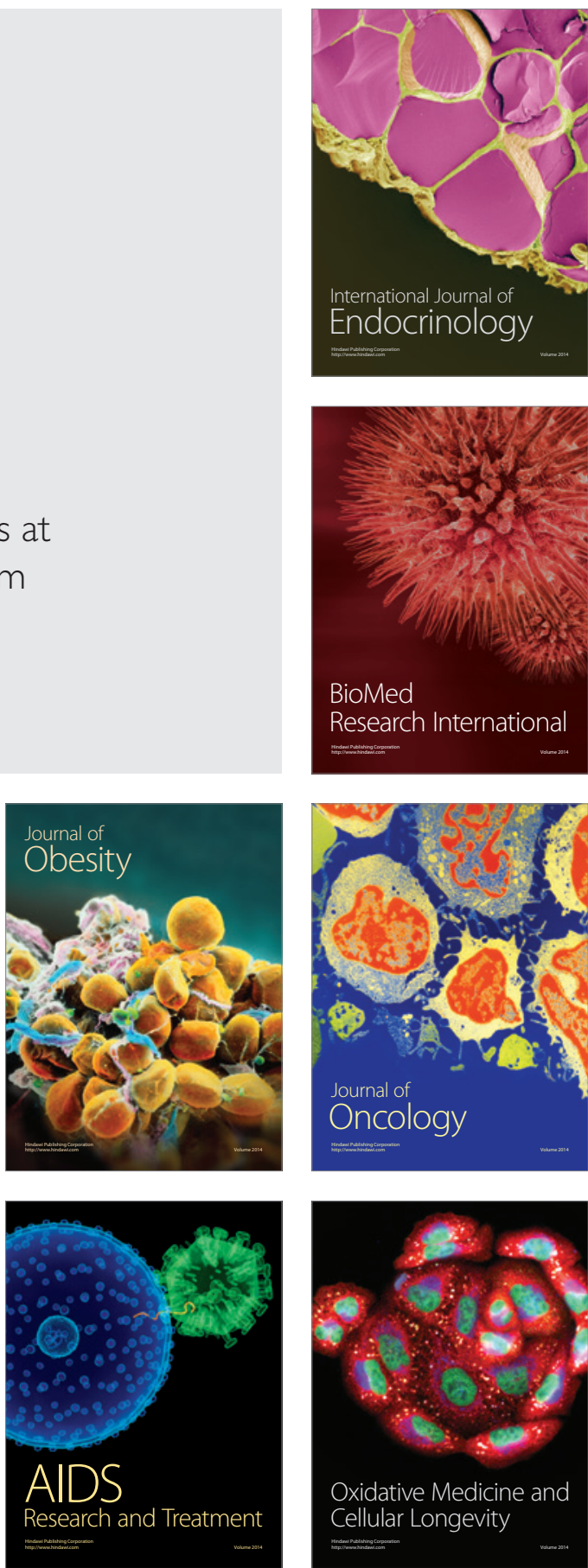